\title{
Insulin's direct effects on the liver dominate the control of hepatic glucose production
}

\author{
Dale S. Edgerton, ${ }^{1}$ Margaret Lautz,, Melanie Scott,, ${ }^{1}$ Carrie A. Everett, ${ }^{1}$ Kathryn M. Stettler, ${ }^{1}$ \\ Doss W. Neal, ${ }^{1}$ Chang A. Chu, ${ }^{2}$ and Alan D. Cherrington ${ }^{1}$
}

1Department of Molecular Physiology and Biophysics, Vanderbilt University School of Medicine, Nashville, Tennessee, USA. ${ }^{2}$ Hurley Consulting Associates Ltd., Chatham, New Jersey, USA.

\begin{abstract}
Insulin inhibits glucose production through both direct and indirect effects on the liver; however, considerable controversy exists regarding the relative importance of these effects. The first aim of this study was to determine which of these processes dominates the acute control of hepatic glucose production (HGP). Somatostatin and portal vein infusions of insulin and glucagon were used to clamp the pancreatic hormones at basal levels in the nondiabetic dog. After a basal sampling period, insulin infusion was switched from the portal vein to a peripheral vein. As a result, the arterial insulin level doubled and the hepatic sinusoidal insulin level was reduced by half. While the arterial plasma FFA level and net hepatic FFA uptake fell by 40-50\%, net hepatic glucose output increased more than 2 -fold and remained elevated compared with that in the control group. The second aim of this study was to determine the effect of a 4-fold rise in head insulin on HGP during peripheral hyperinsulinemia and hepatic insulin deficiency. Sensitivity of the liver was not enhanced by increased insulin delivery to the head. Thus, this study demonstrates that the direct effects of insulin dominate the acute regulation of HGP in the normal dog.
\end{abstract}

\section{Introduction}

Hepatic glucose production (HGP) accounts for the majority of whole-body glucose production and is tightly regulated by insulin in the healthy individual. Since hepatic insulin resistance in diabetic patients results in excess HGP and fasting hyperglycemia (1), it is critical to understand the mechanisms by which insulin regulates this process. Insulin reduces HGP by acting both directly and indirectly on the liver (2); however, there is considerable controversy regarding the relative importance of insulin's direct versus indirect effects under physiological conditions.

Insulin acts directly by binding to hepatic insulin receptors and thereby activating insulin signaling pathways in the liver. These effects have been demonstrated in various models. In isolated rat hepatocytes, insulin inhibits glucose production through inhibition of gluconeogenesis (3) and glycogenolysis (4). In the dog, an acute selective increase (5) or decrease (6) in hepatic insulin level (so that the arterial insulin level was kept constant) resulted in very rapid suppression or stimulation, respectively, of HGP. In addition, liver-specific insulin receptor knockout (LIRKO) mice, which lack hepatic insulin receptors from birth, demonstrate severe hepatic insulin resistance (7). These studies, and others, demonstrate that insulin acts directly on the liver to regulate HGP.

Insulin's indirect effects include reduction of glucagon secretion at the pancreas (8), inhibition of lipolysis in fat (which reduces circulating lipids and glycerol availability for gluconeogenesis) (9), and decreased protein catabolism in muscle (which further reduces gluconeogenic precursor availability) (10), and in addition, recent studies in the mouse and rat suggest that hypothalamic insulin signaling may also play an important role in insulin's ability to indi-

Nonstandard abbreviations used: HGP, hepatic glucose production; ICG, indocyanine green; LIRKO, liver-specific insulin receptor knockout; NHGO, net hepatic glucose output; non-HGU, nonhepatic glucose uptake; Per, peripheral; Por, portal. Conflict of interest: The authors have declared that no conflict of interest exists. Citation for this article: J. Clin. Invest. 116:521-527 (2006). doi:10.1172/JCI27073. rectly regulate $\operatorname{HGP}(11,12)$. Insulin's indirect hepatic effects were well demonstrated in the dog, where an acute selective increase in arterial insulin level (so that the hepatic insulin level was kept constant) resulted in inhibition of HGP (5). This was partially due to insulin's effect on lipolysis, since when plasma FFA levels were prevented from falling, HGP only decreased by half as much (9). In rats, ICV infusion of insulin suppressed glucose production, and this effect was independent of circulating insulin levels (11). In addition, in mice, HGP was suppressed by insulin despite $95 \%$ reduction of hepatic insulin receptors by antisense oligonucleotide treatment (13). These studies, and others, demonstrate that in addition to insulin's direct effects on the liver, the hormone also regulates HGP through indirect means.

Despite evidence demonstrating both direct and indirect effects of insulin on the liver, it has been hypothesized that control of the liver is primarily indirect (14), and a recent study in the mouse questions whether reversal of liver insulin resistance should be a prominent goal of diabetic therapy (15). To address the relative importance of insulin's direct versus indirect effects, a simple experiment was designed to determine which effect dominates control of basal HGP. Experiments were carried out using the overnight-fasted conscious dog, in which, during a pancreatic clamp, insulin infusion was switched from the hepatic portal vein to a peripheral vein. This resulted in a doubling of the arterial insulin level and, at the same time, a 50\% decrease in the insulin level within the hepatic sinusoids. We hypothesized that if HGP is primarily regulated by insulin's indirect effects, then arterial hyperinsulinemia should cause a decrease in HGP, despite a reduction of insulin's direct effect at the liver. On the other hand, if insulin's direct hepatic effects are dominant, increased HGP should occur. In addition, to address data in the rat suggesting the dependence of the regulation of HGP on hypothalamic insulin signaling (11), the second aim of the study was to determine whether an acute 4-fold rise in head insulin level would alter the response to arterial hyperinsulinemia and hepatic insulin deficiency. We hypothesized 

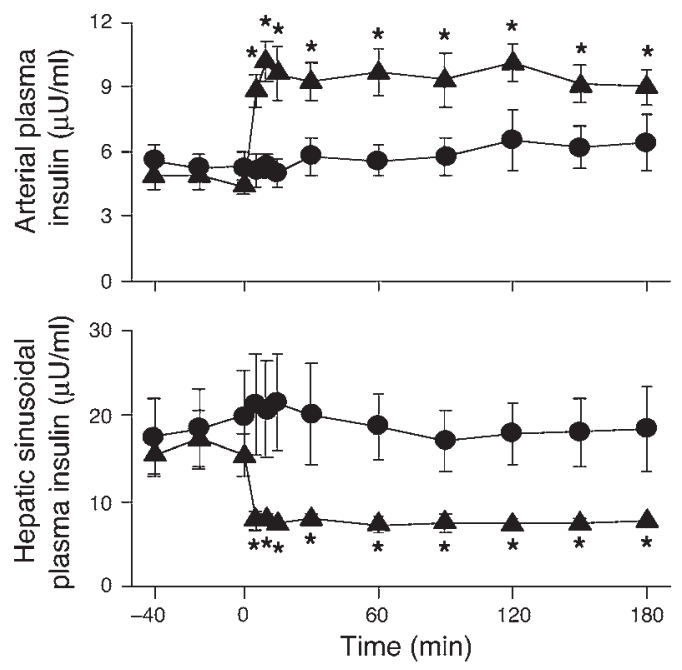

Figure 1

Arterial and hepatic sinusoidal plasma insulin levels in 18-hour-fasted conscious dogs during the basal ( -40 to 0 minutes) and experimental (0-180 minutes) periods. Basal insulin infusion either continued into the portal vein (circles) or was switched to a peripheral vein (triangles) between 0 and 180 minutes. Mean \pm SEM; ${ }^{*} P<0.05$ versus portal group.

that a selective physiological rise in the brain insulin level would suppress HGP through hypothalamic insulin signaling.

\section{Results}

Portal versus peripheral insulin delivery. The arterial and hepatic sinusoidal plasma insulin levels remained basal and unchanged throughout the study in the portal (Por) group (Figure 1). In the peripheral (Per) group, the arterial plasma insulin level rose 2-fold, while the hepatic sinusoidal plasma insulin level decreased by $50 \%$ (Figure 1). The arterial and hepatic sinusoidal glucagon levels remained basal and unchanged in both groups (Figure 2). The arterial plasma FFA level and net hepatic FFA uptake remained unchanged in the Por group but decreased by $40-50 \%$ in the Per group as a result of arterial hyperinsulinemia (Figure 3 ). When the site of insulin delivery was switched from the portal to a peripheral vein in the Per group, net hepatic glucose output (NHGO) increased more than 2-fold, and as a result, the arterial plasma glucose level increased by $30 \%$ (Figure 4). Glucose was infused in the Por group to match this rise in glucose (Table 1), and as a result, NHGO was completely suppressed (Figure 4). Thus, NHGO increased rapidly in the Per group during arterial hyperinsulinemia and hepatic insulin deficiency and, relative to the Por group, remained elevated for the duration of the experimental period. Compared with the Por group, endogenous glucose production was elevated in the Per group, while glucose utilization and clearance were similar (Table 1). Mild hyperglycemia resulted in an increase in nonhepatic glucose uptake (non-HGU) in both groups, although non-HGU tended to be higher in the Per group as a result of the greater arterial insulin levels in that group. The increase in HGP in the Per group was the result of increased net hepatic glycogenolysis, not a change in gluconeogenic flux (Figure 5).

Peripheral versus head insulin delivery. While the head insulin level increased 2-fold in the Per group, there was a 4-fold increase from basal in the head insulin level in the Head group (Figure 6). As in the Per group, the arterial insulin level increased 2-fold in the Head group, and the hepatic sinusoidal insulin level decreased by $50 \%$ (Figure 6). Hepatic sinusoidal glucagon levels were similar in the 2 groups, arterial plasma FFA levels both decreased by approximately $40 \%$, and the arterial plasma glucose levels were matched (Figure 7). Despite the additional rise of the head insulin level in the Head group, NHGO was not suppressed compared with that in the Per group (Figure 8). As in the Per group, endogenous glucose production and non-HGU increased, while there was little change in glucose utilization or clearance (Table 1). Net hepatic glycogenolysis increased in the Head group, while gluconeogenic flux was unaffected (Figure 8).

\section{Discussion}

The first aim of this study was to test whether insulin's direct or indirect effects dominate the control of HGP. When basal insulin delivery was switched from the portal to a peripheral route, the arterial insulin level doubled and the hepatic sinusoidal insulin level was reduced by half. Despite mild hyperglycemia and peripheral hyperinsulinemia, HGP rapidly increased more than 2 -fold and remained elevated relative to that in the Por group throughout the 3-hour experimental period. Therefore, it is clear that insulin's direct effect on the liver was dominant and was the primary controller of basal HGP in the nondiabetic dog. In addition, the increase in arterial insulin level had no effect on wholebody glucose utilization, because increased non-HGU was offset by a decrease in hepatic glucose uptake when insulin infusion was switched from portal vein to peripheral vein delivery.

In a previous study, when the hepatic insulin level was selectively decreased by $75 \%$ and the peripheral insulin level was kept constant, HGP increased 3.75-fold at 15 minutes (6). Following a selective $50 \%$ decrease in hepatic insulin level, a 3 -fold increase in HGP occurred (D.S. Edgerton, unpublished observations; $n=4$ ). In the present study, the $50 \%$ decrease in hepatic insulin and doubling of arterial insulin resulted in a 2-fold increase in HGP at 15
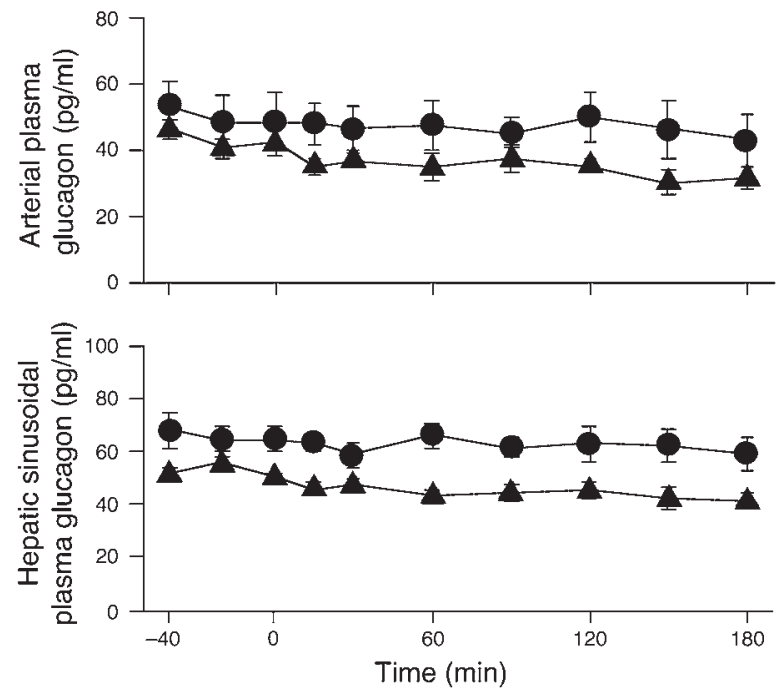

Figure 2

Arterial and hepatic sinusoidal plasma glucagon levels in 18-hour-fasted conscious dogs during the basal ( -40 to 0 minutes) and experimental (0-180 minutes) periods. Basal insulin infusion either continued into the portal vein (circles) or was switched to a peripheral vein (triangles) between 0 and 180 minutes. Mean \pm SEM. 

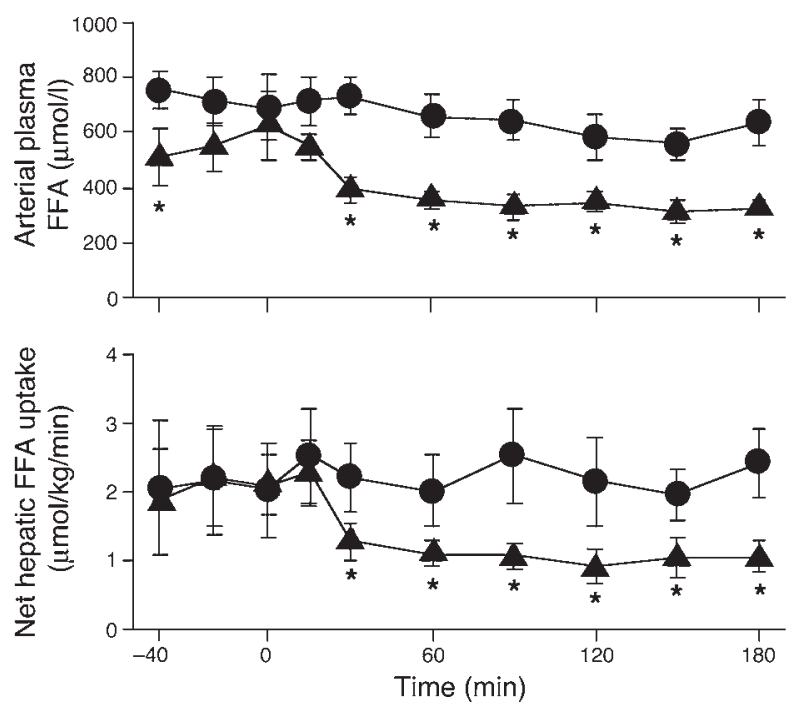

Figure 3

Arterial FFA levels and net hepatic FFA uptake in 18-hour-fasted conscious dogs during the basal ( -40 to 0 minutes) and experimental (0-180 minutes) periods. Basal insulin infusion either continued into the portal vein (circles) or was switched to a peripheral vein (triangles) between 0 and 180 minutes. Mean \pm SEM; ${ }^{*} P<0.05$ versus portal group.

minutes. Thus, although the direct effect of insulin was dominant, the indirect effect suppressed the rise in HGP by about one-third. Much higher peripheral insulin levels would have undoubtedly increased the indirect effect; however, the aim of the study was simply to examine the impact of switching insulin from portal to peripheral delivery, thereby creating a relatively small change in insulin at both sites, to determine which effect would dominate the control of HGP.

In an effort to determine which mode of insulin action is most important in the regulation of HGP, Buettner et al. recently showed that $95 \%$ ablation of hepatic insulin receptors by treatment with insulin receptor antisense oligodeoxynucleotide over 1 week did not impair the ability of insulin to inhibit glucose production during a hyperinsulinemic clamp (13). The conclusion drawn from this study was that hepatic insulin receptors are likely to play an important role in the long-term regulation of HGP, mainly via changes in gene transcription, but rapid control can still occur in the presence of a severe impairment in liver insulin signaling. Thus, as previously demonstrated (5), in the mouse, HGP can be selectively inhibited by insulin's indirect effects, but these findings do not directly address whether insulin's direct or indirect effects dominate the acute control of HGP. Also, although downstream hepatic insulin signaling was blunted in this model, residual hepatic insulin signaling was sufficient to prevent liver pathology or alterations in the expression of insulin-regulated genes and insulin clearance (13). Therefore, the residual insulin signaling may also have been sufficient to mediate direct inhibition of HGP. In addition, although the reduction in the number of hepatic insulin receptors in Buettner et al.'s model was more acute than the lifelong insulin receptor knockout in the LIRKO mouse, it still occurred over a period of 1 week. The present study clearly demonstrates that the acute response of the liver to small changes in hepatic insulin levels occurs within a few minutes, however, and therefore the very rapid response of the liver to the direct effect of insulin is more likely the result of changes in enzyme activity and/or translocation, not gene expression. In addition, after only 3 hours, the mild rise in plasma glucose that accompanied the increase in HGP in the Per group was sufficient to in turn suppress NHGO to the basal rate. Thus, it is difficult to draw a conclusion about acute direct effects of insulin on a liver that has been chronically exposed (i.e., 7 days) to hepatic insulin signaling deficiency (13), when compensatory alterations in the regulation of glucose fluxes are likely to have occurred.

Gupta et al. found that the prevailing glycemic level is a determinant of the balance between insulin's direct and indirect effects on HGP in individuals with type 1 diabetes (16). The authors suggested that when glycogenolysis is already maximally suppressed by hyperglycemia, hepatic insulin may not further suppress HGP, whereas, with correction of hyperglycemia, glycogenolysis is restored, allowing for the direct effect of insulin on glycogenolysis to become manifest. This is in keeping with the results of the present study, which demonstrate that hepatic glycogenolysis, but not gluconeogenesis, is sensitive to acute changes in insulin and glucose. In other studies, obese patients with type 2 diabetes were found to have defects in both the direct and the indirect effects of insulin on the liver (17). When the estimated hepatic sinusoidal insulin levels were increased by $11 \mu \mathrm{U} / \mathrm{ml}$, in those studies, the impairment in the direct effect of insulin was overcome, but the defective indirect response persisted as a result of impaired suppression of FFA and glucagon levels. Thus, while the direct effect of insulin on HGP is dominant in the normal individual, in the diabetic state, insulin resistance of extrahepatic tissues and the prevailing glucose levels become factors.

Previous studies have shown that a 4-fold rise in insulin level throughout the body will completely suppress HGP (18). To further investigate the effect of hypothalamic insulin signaling on HGP, our second aim was to determine the effect of raising the head insulin level on the increased glucose production that
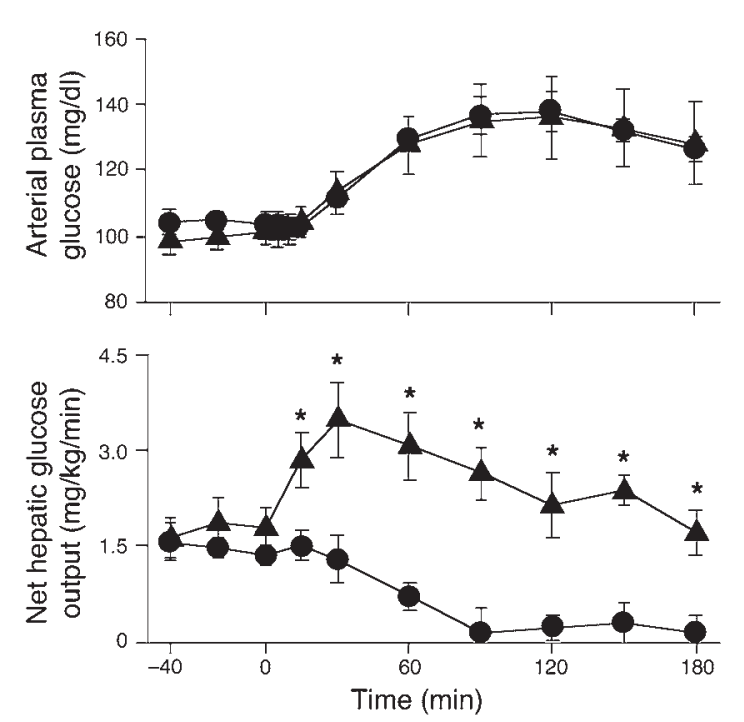

Figure 4

Arterial plasma glucose levels and NHGO in 18-hour-fasted conscious dogs during the basal ( -40 to 0 minutes) and experimental (0-180 minutes) periods. Basal insulin infusion either continued into the portal vein (circles) or was switched to a peripheral vein (triangles) between 0 and 180 minutes. Mean $\pm \mathrm{SEM} ;{ }^{\star} P<0.05$ versus portal group. 


\section{Table 1}

Glucose infusion rate, endogenous glucose production, glucose utilization, glucose clearance, and nonhepatic glucose uptake during the basal ( -40 to 0 minutes) and experimental ( 0 to 180 minutes) periods in the portal, peripheral, and head groups

\begin{tabular}{|c|c|c|c|c|c|c|c|}
\hline Time (min) & Basal period (-40 to 0$)$ & 30 & 60 & 90 & 120 & 150 & 180 \\
\hline \multicolumn{8}{|c|}{ Glucose infusion rate (mg/kg/min) } \\
\hline Portal & $0.00 \pm 0.00$ & $1.19 \pm 0.23$ & $1.88 \pm 0.10$ & $1.82 \pm 0.24$ & $1.40 \pm 0.06$ & $0.82 \pm 0.38$ & $0.62 \pm 0.26$ \\
\hline \multicolumn{8}{|c|}{ Endogenous glucose production (mg/kg/min) } \\
\hline Portal & $2.70 \pm 0.61$ & $2.53 \pm 0.64$ & $1.83 \pm 0.62$ & $1.64 \pm 0.67$ & $1.47 \pm 0.49$ & $1.36 \pm 0.34$ & $1.11 \pm 0.35$ \\
\hline Peripheral & $2.75 \pm 0.21$ & $3.73 \pm 0.25^{A}$ & $4.10 \pm 0.22^{\mathrm{A}}$ & $3.46 \pm 0.36^{A}$ & $3.03 \pm 0.40^{A}$ & $2.66 \pm 0.40^{\mathrm{A}}$ & $2.35 \pm 0.28^{A}$ \\
\hline Head & $2.53 \pm 0.24$ & $4.37 \pm 0.58^{A}$ & $3.86 \pm 0.50^{A}$ & $2.97 \pm 0.26 \mathrm{~A}$ & $2.83 \pm 0.31^{A}$ & $3.04 \pm 0.36^{A}$ & $3.01 \pm 0.40^{A}$ \\
\hline \multicolumn{8}{|c|}{ Glucose utilization (mg/kg/min) } \\
\hline Portal & $2.71 \pm 0.50$ & $2.95 \pm 0.53$ & $2.72 \pm 0.48$ & $3.00 \pm 0.44$ & $3.04 \pm 0.35$ & $2.79 \pm 0.23$ & $2.45 \pm 0.23$ \\
\hline Peripheral & $2.62 \pm 0.24$ & $2.51 \pm 0.35$ & $2.78 \pm 0.36$ & $2.73 \pm 0.44$ & $3.05 \pm 0.44$ & $3.02 \pm 0.36$ & $2.80 \pm 0.27$ \\
\hline Head & $2.70 \pm 0.28$ & $2.97 \pm 0.30$ & $3.35 \pm 0.45$ & $3.11 \pm 0.29$ & $3.12 \pm 0.31$ & $3.38 \pm 0.41$ & $3.30 \pm 0.42$ \\
\hline \multicolumn{8}{|c|}{ Glucose clearance (ml/kg/min) } \\
\hline Portal & $2.63 \pm 0.51$ & $2.62 \pm 0.50$ & $2.13 \pm 0.33$ & $2.11 \pm 0.33$ & $2.15 \pm 0.23$ & $2.04 \pm 0.14$ & $2.02 \pm 0.13$ \\
\hline Peripheral & $2.46 \pm 0.37$ & $2.15 \pm 0.43$ & $2.15 \pm 0.45$ & $2.03 \pm 0.47$ & $2.28 \pm 0.56$ & $2.32 \pm 0.56$ & $2.23 \pm 0.51$ \\
\hline Head & $2.47 \pm 0.23$ & $2.24 \pm 0.15$ & $2.32 \pm 0.22$ & $2.17 \pm 0.14$ & $2.25 \pm 0.19$ & $2.22 \pm 0.46$ & $2.50 \pm 0.22$ \\
\hline \multicolumn{8}{|c|}{ Nonhepatic glucose uptake $(\mathrm{mg} / \mathrm{kg} / \mathrm{min})$} \\
\hline Portal & $1.48 \pm 0.20$ & $3.05 \pm 0.46$ & $3.25 \pm 0.13$ & $2.23 \pm 0.33$ & $1.67 \pm 0.26$ & $0.91 \pm 0.63$ & $0.56 \pm 0.37$ \\
\hline Peripheral & $1.84 \pm 0.35$ & $4.22 \pm 0.95$ & $3.66 \pm 0.63$ & $2.84 \pm 0.56$ & $2.24 \pm 0.56$ & $2.19 \pm 0.32$ & $1.63 \pm 0.39$ \\
\hline Head & $1.33 \pm 0.24$ & $4.12 \pm 1.09$ & $2.94 \pm 0.55$ & $1.99 \pm 0.48$ & $1.49 \pm 0.20$ & $1.18 \pm 0.37$ & $1.11 \pm 0.28$ \\
\hline
\end{tabular}

Values are means $\pm \mathrm{SEM} ; n=5,6$, and 6 per group, respectively; ${ }^{\mathrm{A} P}<0.05$

was seen in response to hepatic insulin deficiency. A switch from portal insulin infusion to head artery delivery (at the same basal rate) resulted in a 4-fold increase in head insulin level, relative to the basal insulin level. Compared with the Per group, this elevation did not bring about an additional inhibitory effect on the liver, however.

In studies in the rat, Obici et al. showed that ICV infusion of insulin resulted in increased hepatic insulin sensitivity (so that glucose production fell during a euinsulinemic pancreatic clamp during ICV insulin infusion) (11). In addition, when insulin receptor antisense oligonucleotide, insulin antibodies, or PI3K inhibitors were infused into the third ventricle, glucose production was not suppressed by a rise in arterial insulin as much as in a control group $(23 \%, 10 \%$, and $34 \%$ less than what would be predicted based on the relative increase in insulin in the 3 groups respectively) (12). These findings led the authors to conclude that hypothalamic insulin signaling is required for normal inhibition of glucose production by insulin (11).

One potential difference between the Obici et al. study and the present study is that hypothalamic insulin levels may have been higher in the rat than in the dog. The amount of insulin injected ICV in the rat was $30 \mu \mathrm{U}$, and since the volume of distribution of insulin in these studies is unknown and likely to be very small, there was potentially a much higher local concentration of insulin. On the other hand, in the dog the increase in insulin level in blood entering the head was only 4-fold compared with the basal level, so it is difficult to know what difference in hypothalamic insulin

\section{Figure 5}

Net hepatic glycogenolysis and hepatic gluconeogenic flux in 18hour-fasted conscious dogs during the basal ( -40 to 0 minutes) and experimental (0-180 minutes) periods. Basal insulin infusion either continued into the portal vein (circles) or was switched to a peripheral vein (triangles) between 0 and 180 minutes. Mean \pm SEM; ${ }^{*} P<0.05$ versus portal group. exposure there may have been. The blood-brain barrier is permeable to insulin (19), and previous studies in the dog have demonstrated that infusion into the carotid and vertebral arteries results in even distribution throughout the blood flowing through the head (20). In addition, a rapid change in sympathetic outflow from the brain was previously demonstrated in response to head insulin infusion in the $\operatorname{dog}(21)$. These studies suggest that the head arterial insulin infusion model used in this study is appropriate for the assessment of the effects of a physiological rise of insulin on HGP. Although it is possible that the 3-hour experimental period did not allow enough time for sufficient amounts of insulin to reach the hypothalamus compared with the 6-hour ICV insulin infusion in the rat, previous studies in the dog demonstrated that cerebrospinal fluid insulin levels increase (after a 30-minute lag) during a hyperinsulinemic clamp (22). In that study, when the plasma
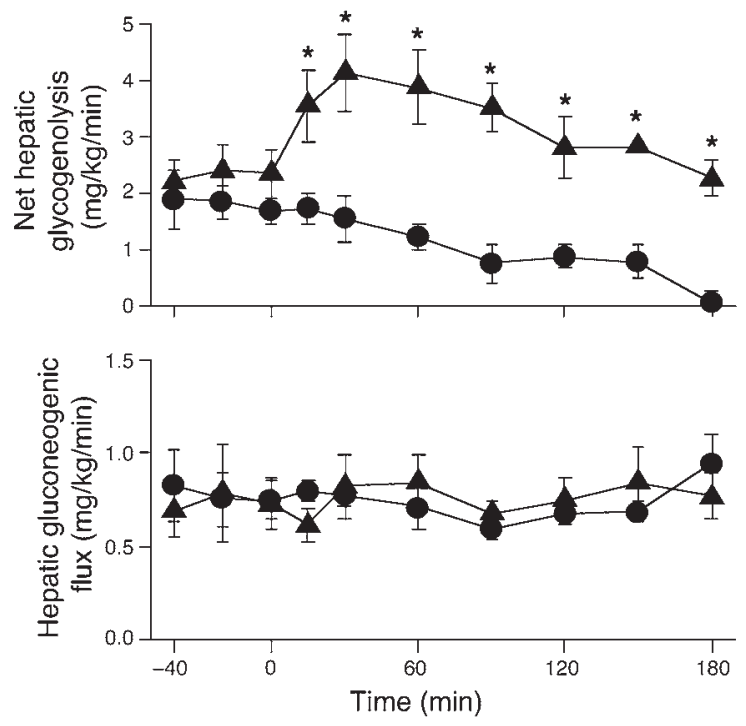


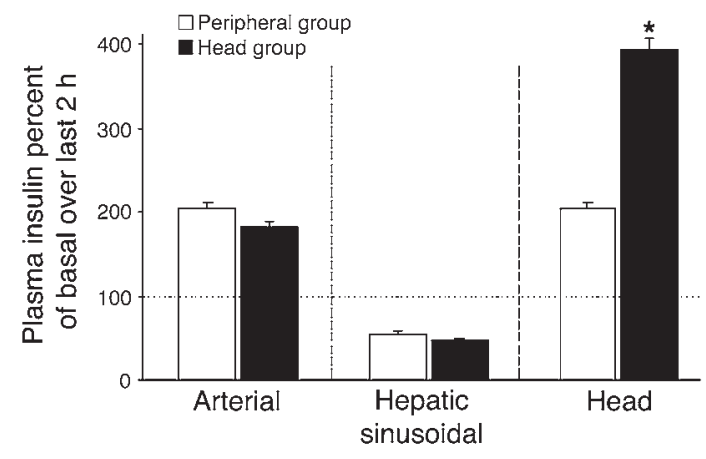

insulin level was clamped at approximately $90 \mu \mathrm{U} / \mathrm{ml}$, cerebrospinal fluid insulin was approximately $2 \mu \mathrm{U} / \mathrm{ml}$ after 180 minutes. Therefore the insulin concentration in the cerebrospinal fluid in the present study was probably much lower. If a 4-fold, 3-hour rise in insulin is not sufficient to cause a change in insulin signaling in the hypothalamus, however, then this process is unlikely to play a role in the acute regulation of HGP.

Another factor to consider is the considerably higher basal glucose production in rats $(\sim 13 \mathrm{mg} / \mathrm{kg} / \mathrm{min})$ compared with dogs and humans $(\sim 3 \mathrm{mg} / \mathrm{kg} / \mathrm{min})$, which may be the result of higher neural drive to the liver. If this is the case, it is likely that brain insulin levels would exert greater control of glucose production in the rat compared with species with lower basal glucose production rates. Finally, the rat studies were carried out in the presence of basal peripheral insulin levels, whereas hepatic insulin deficiency occurred in the dog. Hepatic insulin signaling was presumably present but reduced in this study; therefore it is possible that the effect of insulin in the brain is manifest only in the presence of normal hepatic insulin signaling, since findings in the LIRKO model suggest that insulin signaling in the liver is required for the indirect effects of insulin (7).

Okamoto et al. recently demonstrated hepatic insulin resistance in insulin receptor knockout mice with restored liver insulin signaling, but reduced signaling in the hypothalamus (15). This suggests that hypothalamic signaling plays an important role in insulin's ability to regulate HGP in the mouse. If this finding applies to the dog, since HGP was not acutely reduced during head hyperinsulinemia in the present study, hypothalamic insulin signaling may play a greater role in the chronic regulation of HGP, acting to set the basal tone for insulin sensitivity at the liver. Thus, background hypothalamic insulin signaling may determine the acute ability of the liver to directly respond to insulin, but not be involved in the

\section{Figure 7}

Percentage of hepatic sinusoidal glucagon and arterial plasma FFA and glucose with respect to basal levels during the last 2 hours in 18hour-fasted conscious dogs. Basal insulin infusion was switched from the portal vein either to a peripheral vein (white bars) or to head arteries (black bars) between 0 and 180 minutes. Mean \pm SEM.

\section{Figure 6}

Percentage of arterial, hepatic sinusoidal, and head plasma insulin with respect to basal levels during the last 2 hours in 18-hour-fasted conscious dogs. Basal insulin infusion was switched from the portal vein either to a peripheral vein (white bars) or to head arteries (black bars) between 0 and 180 minutes. Mean $\pm \mathrm{SEM}$; ${ }^{*} P<0.05$ versus portal group. minute-to-minute regulation of HGP. Although the rodent studies mentioned above provide strong support for the involvement of hypothalamic insulin signaling in the regulation of HGP, further studies are required to determine how these findings will extend to other species. Again, if the rates of basal glucose production in the mouse as reported by the authors $(29 \mathrm{mg} / \mathrm{kg} / \mathrm{min})$ are an indication of high constitutive neural drive to the liver, this would be expected to make the role of the brain more important in the control of HGP in the absence of hepatic insulin receptors.

The present study was designed to determine whether insulin's direct or indirect effects dominate the regulation of HGP. The results clearly demonstrate the primary importance of insulin's direct effect on the acute regulation of basal HGP, since when this effect was reduced by $50 \%$ there was a rapid increase in HGP, despite simultaneous arterial hyperinsulinemia and hyperglycemia and an associated reduction in plasma FFA and net hepatic FFA uptake. In addition, sensitivity of the liver to insulin was not enhanced by increased insulin delivery to the head, calling into question the role of hypothalamic insulin signaling in the acute control of basal HGP in species in which basal glucose production is low.

\section{Methods}

Animal care and surgical procedures. Experiments were conducted on 3 groups (Por, Per, and Head; $n=5,6$, and 6 respectively) of overnight-fasted conscious mongrel dogs (20-28 kg) of either sex. Housing and diet have been described previously (23). The surgical facility met the standards published by the American Association for the Accreditation of Laboratory Animal Care, and the protocols were approved by the Vanderbilt University Medical Center Animal Care Committee. All dogs underwent a laparotomy 2 weeks before the experiment in order to implant infusion catheters into the jejunal and splenic veins, sampling catheters into the portal and hepatic veins and the femoral artery, and Transonic flow probes (Transonic Sys- 


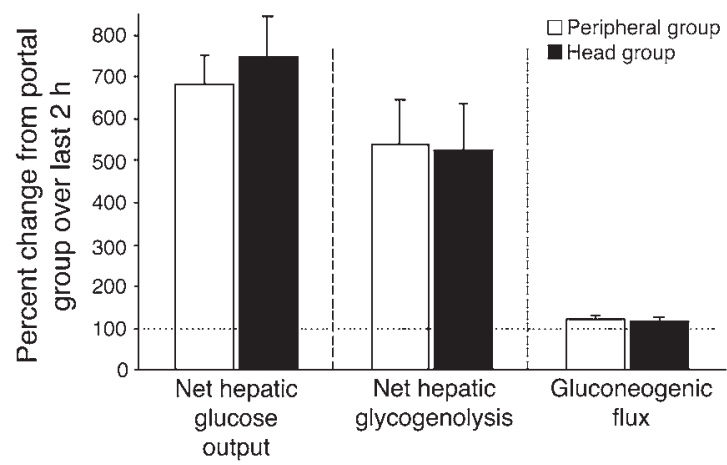

tems Inc.) around the hepatic artery and portal vein, as described elsewhere (23). In the Head group, a second surgery was performed 8-9 days before each experiment. A ventral midline incision was made under general anesthesia, and Silastic catheters (Dow Corning) were inserted into the vertebral and carotid arteries bilaterally (20). A catheter was also inserted into the left jugular vein to allow blood sampling so that the head insulin level could be monitored. Each dog was used for only 1 experiment. All dogs studied were healthy, as indicated by (a) a leukocyte count less than 18,000 per cubic millimeter, (b) hematocrit greater than $35 \%$, (c) a good appetite, and (d) normal stools.

Experimental design. Intraportal catheters (splenic and jejunal) were used for the infusion of insulin (Eli Lilly and Co.) and glucagon (Novo Nordisk). Angiocaths (Deseret Medical, BD) were inserted percutaneously into leg veins for infusion of $\left[3-{ }^{3} \mathrm{H}\right]$ glucose (DuPont-NEN), indocyanine green (ICG; Sigma-Aldrich), and peripheral glucose (50\% dextrose; Baxter International Inc.). Animals were allowed to rest quietly in a Pavlov harness for 30 minutes before the experiments started. Each of the 3 protocols consisted of an equilibration period ( -140 to -40 minutes), a basal period ( -40 to 0 minutes), and an experimental period (0 to 180 minutes). At -140 minutes a priming dose of $\left[3-{ }^{3} \mathrm{H}\right]$ glucose $(50 \mu \mathrm{Ci})$ was given, and constant infusions of $\left[3-{ }^{3} \mathrm{H}\right]$ glucose $(0.4 \mu \mathrm{Ci} / \mathrm{min})$ and ICG $(0.07 \mathrm{mg} / \mathrm{min})$ were started. At the same time a constant infusion of somatostatin $(0.8 \mu \mathrm{g} / \mathrm{kg} / \mathrm{min})$ was started in a peripheral vein to inhibit endogenous pancreatic hormone secretion, and a constant intraportal glucagon infusion $(0.5 \mathrm{ng} / \mathrm{kg} / \mathrm{min})$ was started to replace basal secretion of this hormone. Plasma glucose samples were taken every 5 minutes, and the intraportal insulin infusion rate was titrated as necessary to maintain glucose at basal levels. The last change was made at least 20 minutes before the start of the control period. The insulin infusion rates were $211 \pm 39,200 \pm 21$, and $223 \pm 27 \mu \mathrm{U} / \mathrm{kg} / \mathrm{min}$ in the Por, Per, and Head groups, respectively. After the basal period, the portal insulin infusion was continued in the Por group. In the Per group the insulin infusion was switched from hepatic portal to peripheral vein delivery, and in the Head group it was switched to the carotid and vertebral arteries. Immediately after the final sampling time, each animal was euthanized and the positions of the catheter tips were verified.

Analytical procedures. Hematocrit concentrations; plasma glucose, $\left[{ }^{3} \mathrm{H}\right]$ glucose, glucagon, insulin, cortisol, and FFA concentrations; and blood alanine, glycine, serine, threonine, lactate, glutamine, glutamate, glycerol, $\beta$-hydroxybutyrate, and acetoacetate concentrations were determined as previously described $(23,24)$.

Calculations. Net hepatic balances (NHBs) were calculated with the arterio-venous difference method using the formula: $\mathrm{NHB}=$ Load $_{\text {out }}-$ Load $_{\text {in }}$, where $\operatorname{Load}_{\text {out }}=[\mathrm{H}] \times \mathrm{HF}$ and $\operatorname{Load}_{\text {in }}=[\mathrm{A}] \times \mathrm{AF}+[\mathrm{P}] \times \mathrm{PF}$ and where $[\mathrm{H}]$, $[\mathrm{A}]$, and $[\mathrm{P}]$ are the substrate concentrations in hepatic vein, femoral artery, and portal vein blood or plasma, respectively, and $\mathrm{HF}, \mathrm{AF}$, and $\mathrm{PF}$ are the blood flow in the hepatic vein, hepatic artery, and portal vein, respectively,

\section{Figure 8}

Percentage change in net hepatic glucose output and glycogenolysis and gluconeogenic flux compared with those in the portal group during the last 2 hours in 18-hour-fasted conscious dogs. Basal insulin infusion was switched from the portal vein either to a peripheral vein (white bars) or to head arteries (black bars) between 0 and 180 minutes. Mean \pm SEM.

as determined by the ultrasonic flow probes. Hepatic blood flow was also determined from ICG extraction across the liver to verify the ultrasonic measurements. A positive hepatic balance value represents net output by the liver, while a negative value represents net hepatic uptake. Plasma glucose and $\left[{ }^{3} \mathrm{H}\right]$ glucose values were multiplied by 0.73 to convert them to blood glucose values as validated elsewhere (25). Tracer-determined wholebody glucose appearance and utilization were measured using a primed, constant infusion of $\left[3-{ }^{3} \mathrm{H}\right]$ glucose. Data calculation was carried out using the 2-compartment model described by Mari (26) and with canine parameters reported by Dobbins et al. (27). Endogenous glucose appearance was calculated by subtraction of the glucose infusion rate from whole-body glucose appearance. Non-HGU was calculated as the glucose infusion rate plus net hepatic glucose balance, with changes in the glucose mass accounted for when deviations from steady state were present. The approximate insulin and glucagon levels in plasma entering the liver sinusoids were calculated using the formula $[\mathrm{A}] \times \% \mathrm{AF}+[\mathrm{P}] \times \% \mathrm{PF}$, where $[\mathrm{A}]$ and $[\mathrm{P}]$ are arterial and portal vein hormone concentrations, respectively, and \% $\mathrm{AF}$ and $\% \mathrm{PF}$ are the respective percentage contributions of arterial and portal flow to total hepatic blood flow. Gluconeogenic flux and net hepatic glycogenolysis were determined using the arterio-venous difference method.

Statistics. The data were analyzed for differences from the basal period and for differences from the control group. Statistical comparisons were carried out using 2-way repeated-measures ANOVA (SigmaStat; SPSS Inc.). One-way ANOVA comparison tests were used post hoc when significant $F$ ratios were obtained. Significance was established when $P$ was less than 0.05 (2-sided test).

\section{Acknowledgments}

This research was supported by NIH grant R37 DK18243 and an American Diabetes Association Mentor-Based Postdoctoral Fellowship grant to Alan D. Cherrington. We wish to thank Jon Hastings, Angelina Penaloza, Wanda Snead, and Patrick Donahue for their excellent technical support.

Received for publication October 6, 2005, and accepted in revised form November 15, 2005.

Address correspondence to: Dale Edgerton, Department of Molecular Physiology and Biophysics, Vanderbilt University School of Medicine, 710 Robinson Building, 2200 Pierce Avenue, Nashville, Tennessee 37232, USA. Phone: (615) 322-7014; Fax: (615) 3221462; E-mail: dale.edgerton@vanderbilt.edu.

This work was presented in part at the 65th Annual Meeting of the American Diabetes Association in San Diego, California, USA, in June 2005. 
1. DeFronzo, R.A., Bonadonna, R.C., and Ferrannini, E. 1992. Pathogenesis of NIDDM. A balanced overview. Diabetes Care. 15:318-368.

2. Cherrington, A.D., Edgerton, D., and Sindelar, D.K. 1998. The direct and indirect effects of insulin on hepatic glucose production in vivo. Diabetologia. 41:987-996.

3. Claus, T.H., and Pilkis, S.J. 1976. Regulation by insulin of gluconeogenesis in isolated rat hepatocytes. Biochim. Biophys. Acta. 421:246-262.

4. Marks, J.S., and Botelho, L.H. 1986. Synergistic inhibition of glucagon-induced effects on hepatic glucose metabolism in the presence of insulin and a cAMP antagonist. J. Biol. Chem. 261:15895-15899.

5. Sindelar, D.K., Balcom, J.H., Chu, C.A., Neal, D.W., and Cherrington, A.D. 1996. A comparison of the effects of selective increases in peripheral or portal insulin on hepatic glucose production in the conscious dog. Diabetes. 45:1594-1604.

6. Sindelar, D.K., et al. 1998. Basal hepatic glucose production is regulated by the portal vein insulin concentration. Diabetes. 47:523-529.

7. Fisher, S.J., and Kahn, C.R. 2003. Insulin signaling is required for insulin's direct and indirect action on hepatic glucose production. J. Clin. Invest. 111:463-468. doi:10.1172/JCI200316426.

8. Ito, K., et al. 1995. Exogenous insulin dose-dependently suppresses glucopenia-induced glucagon secretion from perfused rat pancreas. Metabolism. 44:358-362.

9. Sindelar, D.K., et al. 1997. The role of fatty acids in mediating the effects of peripheral insulin on hepatic glucose production in the conscious dog.
Diabetes. 46:187-196.

10. Wolfe, R.R., and Volpi, E. 2001. Insulin and protein metabolism. In Handbook of physiology: the endocrine pancreas and regulation of metabolism. L.S. Jefferson and A. Cherrington, editors. Oxford University Press. New York, New York, USA. 735-757.

11. Obici, S., Zhang, B.B., Karkanias, G., and Rossetti, L. 2002. Hypothalamic insulin signaling is required for inhibition of glucose production. Nat. Med. 8:1376-1382.

12. Obici, S., Feng, Z., Karkanias, G., Baskin, D.G., and Rossetti, L. 2002. Decreasing hypothalamic insulin receptors causes hyperphagia and insulin resistance in rats. Nat. Neurosci. 5:566-572.

13. Buettner, C., et al. 2005. Severe impairment in liver insulin signaling fails to alter hepatic insulin action in conscious mice. J. Clin. Invest. 115:1306-1313. doi:10.1172/JCI200523109.

14. Bergman, R.N. 2000. Non-esterified fatty acids and the liver: why is insulin secreted into the portal vein? Diabetologia. 43:946-952.

15. Okamoto, H., Obici, S., Accili, D., and Rossetti, L. 2005. Restoration of liver insulin signaling in Insr knockout mice fails to normalize hepatic insulin action. J. Clin. Invest. 115:1314-1322. doi:10.1172/ JCI200523096.

16. Gupta, N., et al. 2002. Insulin inhibits glucose production by a direct effect in diabetic depancreatized dogs during euglycemia. Am. J. Physiol. Endocrinol. Metab. 283:E1002-E1007.

17. Staehr, P., Hother-Nielsen, O., Levin, K., Holst, J.J., and Beck-Nielsen, H. 2001. Assessment of hepatic insulin action in obese type 2 diabetic patients. Dia- betes. 50:1363-1370

18. Steiner, K.E., Williams, P.E., Lacy, W.W., and Cherrington, A.D. 1990. Effects of insulin on glucagonstimulated glucose production in the conscious dog. Metabolism. 39:1325-1333.

19. Woods, S.C., Seeley, R.J., Baskin, D.G., and Schwartz, M.W. 2003. Insulin and the blood-brain barrier. Curr. Pharm. Des. 9:795-800.

20. Biggers, D.W., et al. 1989. Role of brain in counterregulation of insulin-induced hypoglycemia in dogs. Diabetes. 38:7-16.

21. Davis, S.N., et al. 1995. Evidence that the brain of the conscious dog is insulin sensitive. J. Clin. Invest. 95:593-602.

22. Schwartz, M.W., et al. 1990. Kinetics and specificity of insulin uptake from plasma into cerebrospinal fluid. Am. J. Physiol. 259:E378-E383.

23. Edgerton, D.S., et al. 2001. Small increases in insulin inhibit hepatic glucose production solely caused by an effect on glycogen metabolism. Diabetes. 50:1872-1882.

24. Price, C.P., Llyod, B., and Alberti, G.M. 1977. A kinetic spectrophotometric assay for rapid determination of acetoacetate in blood. Clin. Chem. 23:1893-1897.

25. Moore, M.C., et al. 1991. Sources of carbon for hepatic glycogen synthesis in the conscious dog. J. Clin. Invest. 88:578-587.

26. Mari, A. 1992. Estimation of the rate of appearance in the non-steady state with a two-compartment model. Am. J. Physiol. 263:E400-E415.

27. Dobbins, R.L., et al. 1994. Pulsatility does not alter the response to a physiological increment in glucagon in the conscious dog. Am. J. Physiol. 266:E467-E478. 